\title{
Stroke prevention
}

\author{
R Tozer, IR Hastie
}

Although there have been considerable improvements in the care of patients following stroke, there are still no drug treatments which have been shown to conclusively improve recovery. Trials still underway are looking at changes in outcome using drugs aimed at limiting the size of the ischaemic penumbra, but there are already a number of proven strategies for prevention of stroke. Programmes to reduce smoking, alcohol intake, and obesity, to increase exercise and to improve glycaemic control in diabetics are no longer controversial, and a decrease in stroke incidence is only one of the expected health benefits of these measures, but the remainder of this article will deal with the options for drug and surgical treatment in the prevention of stroke.

\section{Hypertension}

Hypertension has long been recognised as an independent risk factor for stroke, and effective control will reduce the rate of stroke, a finding which has recently been confirmed in three large trials in elderly subjects. ${ }^{1-3}$ The STOPHypertension trial recruited patients aged $70-84$ years with systolic pressure of $180-230 \mathrm{mmHg}$ and diastolic pressure of at least $90 \mathrm{mmHg}$, or diastolic pressure of $105-120 \mathrm{mmHg}$ alone. Significant reductions were achieved in all primary end-points, including fatal and non-fatal stroke, and total mortality. There was a reduction of 14.5 strokes per 1000 patient-years on treatment. The MRC trial studied people aged 65-74 years with mean systolic pressures of 160-209 $\mathrm{mmHg}$ and mean diastolic pressures $<115 \mathrm{mmHg}$. Treatment, particularly in the sub group of smokers treated with diuretics, reduced stroke, coronary and all cardiovascular events, reducing the number of strokes by 2.7 per 1000 patient-years of treatment. The SHEP study of isolated systolic hypertension (160-219 $\mathrm{mmHg}$ with diastolic pressure $<90 \mathrm{mmHg}$ ) in subjects over 60 years old showed a reduction in stroke, cardiovascular and coronary morbidity and mortality, and in overall mortality. Six strokes (mainly non-fatal) were prevented per 1000 patient-years of treatment. It appears that isolated systolic hypertension should be treated in the same way as diastolic hypertension, although this has only been demonstrated in the highly selected group of elderly subjects recruited to the SHEP study so far.

Treatment of hypertension in elderly people therefore prevents stroke, but the cost in terms of patient years on treatment may be considerable. This cost may be even greater in younger patients. The MRC trial of treatment of mild hypertension under the age of 65 years required 850 patient years of treatment to prevent one stroke. ${ }^{4}$ This situation might be improved by concentrating treatment on subgroups of hypertensive patients with high risk of stroke, an approach highlighted by follow-up analysis of the MRC trial. ${ }^{5}$ Patients with high blood pressure but no other risk factors may have a low total stroke risk. Treating patients on the basis of overall risk rather than on an arbitrary level of blood pressure is an approach supported by the epidemiological evidence, ${ }^{6}$ and is likely to prove to be a more cost effective approach to stroke prevention. The relationship between rate of cardiovascular complications and level of systolic or diastolic blood pressure is linear, ie, there is no safe upper limit for blood pressure, and patients with a high stroke risk may benefit from having even relatively low blood pressure reduced further.

\section{Primary prevention in patients with atrial fibrillation}

Atrial fibrillation complicating rheumatic mitral valve disease has long been recognised as an important cause of embolic stroke, and has been seen as an indication for anticoagulation, although there are no placebo-controlled trials to confirm the effectiveness of this approach. Evidence from the Framingham study has shown that stroke is more common in patients with atrial fibrillation whatever the cause, ${ }^{7}$ and that it is an independent risk factor for stroke. ${ }^{8}$ The risk of ischaemic stroke is five times as great for patients with non-valvular atrial fibrillation compared with the general population, ${ }^{9}$ and is four times as great for

Division of Geriatric Medicine, St George's Hospital Medical School, R Tozer

IR Hastie

Accepted 27 June 1994 
patients with lone atrial fibrillation, ie, without other evidence of heart disease. ${ }^{10}$ However this risk may be much lower for patients with lone atrial fibrillation under the age of 65 years. ${ }^{11}$ The long held view that patients with paroxysmal atrial fibrillation have a particularly high risk of stroke does not appear to be the case, with a risk of stroke approximately twice that of the general population. ${ }^{12}$ However there does appear to be a clustering of embolic episodes in the first months after conversion from paroxysmal to chronic atrial fibrillation. ${ }^{12}$

If atrial fibrillation is a risk factor for embolic stroke, the risk of stroke might be expected to be reduced by anticoagulation. This has now been shown to be the case in five multicentre trials of primary prevention with warfarin ${ }^{13-17}$ and in one trial of warfarin as secondary prevention in patients with atrial fibrillation after transient ischaemic attack or non-disabling stroke. ${ }^{18}$ The target level of anticoagulation varied between the five trials, but it appears that achieving a prothrombin-time ratio of 1.2 to 1.5 (equivalent to an international normalised ratio (INR) of approximately 1.5 to 3 ) reduces stroke risk by $69 \%$, equivalent to preventing approximately 30 strokes per 1000 patient years on treatment. The complication rate was low in all the trials, including a rate for intracranial haemorrhage of less than $0.5 \%$ per year. These results are probably applicable to elderly patients as the trials included a significant number of patients over 75 years.

The role of aspirin in primary prevention of stroke in patients with atrial fibrillation has also been under recent investigation. Two of the above trials used aspirin or placebo as an alternative to anticoagulation. While one showed a significant reduction in cardio-embolic events using $325 \mathrm{mg}$ of aspirin daily in patients under 75 years, ${ }^{15}$ the other showed a non-significant reduction with $75 \mathrm{mg}$ daily. ${ }^{13}$ The second Stroke Prevention in Atrial Fibrillation (SPAF II) trial has recently reported an equal reduction in stroke with $325 \mathrm{mg}$ aspirin when compared with anticoagulation to achieve an INR of 2.0-4.5 in 1100 patients. ${ }^{19}$

It would seem reasonable to advise aspirin treatment to those patients in atrial fibrillation who have a low overall risk of stroke (ie, in the absence of hypertension, previous thrombo-embolism or recent heart failure, and with a normal echocardiogram) or in whom anticoagulation is contraindicated, and warfarin for high risk patients. This approach needs to be tested by further trials, however. Age alone should not be used as a contraindication to anticoagulation as although there are good theoretical reasons for expecting a greater risk of haemorrhagic complications with increasing age, the trial evidence is contradictory. ${ }^{20-23}$

\section{Secondary prevention}

Stroke may be preceded by transient ischaemic attacks. The Oxfordshire Community Stroke Project has shown a 13-fold excess risk of stroke in the first year after a transient ischaemic attack and a sevenfold excess risk over the first seven years compared with controls. ${ }^{24}$ Aspirin treatment is of proven benefit in reducing subsequent stroke after both transient ischaemic attack and nondisabling embolic stroke. ${ }^{25}$ The dose of aspirin used appears to make little difference to outcome, but $75 \mathrm{mg}$ daily would be expected to have a lower incidence of side effects than higher doses.

Carotid endarterectomy has been used extensively to treat patients with carotid stenosis, especially in the US, in the belief that surgery reduces the risk of stroke. This has now been confirmed for symptomatic patients with severe $(>70 \%)$ stenosis. ${ }^{26,27}$ The risk of arteriography and surgery outweigh the benefits for mild stenosis $(<30 \%)$ and the trials are continuing to look at patients with moderate $(30-70 \%)$ disease. The place of surgery in asymptomatic patients is also awaiting the outcome of trials still in progress. ${ }^{28,29}$ The benefits of surgery must be weighed against the risks which rise with age, and age is bound to be a factor in the decision to offer surgery. The MRC trial had no upper age limit for inclusion, but age would certainly have been a factor affecting referral into the study. The mean age of patients with severe stenosis was 62.2 years. The North American Symptomatic Carotid Endarterectomy Trial had an upper age limit of 80 years with a median age of 65 years for the group undergoing surgery. The mortality and morbidity of endarterectomy would be expected to be low in the highly motivated centres performing many operations per year which take part in multicentre trials. Only if these low levels of risk can be replicated at a local level can surgery be recommended for individual patients. This is a powerful argument for organisation of vascular surgery services into a relatively small number of centres, each performing a large number of operations.

The place of anticoagulation or aspirin in secondary prevention after a transient ischaemic attack or non-disabling stroke in patients with atrial fibrillation has recently been addressed. ${ }^{18}$ These patients have a high risk of 


\section{Stroke prevention}

- strokes are not inevitable, some can be prevented

- results of trials of treatments in acute stroke to reduce cerebral ischaemia will soon be available

- control of hypertension can reduce the incidence of stroke even in the very old

- the use of warfarin should be seriously considered in an older person with atrial fibrillation

- transient ischaemic attacks give warning of a greatly increased risk of stroke

- endarterectomy to prevent stroke should be considered if $>70 \%$ of carotid stenosis present

subsequent stroke with an annual rate of $12 \%$ which was reduced to $4 \%$ by anticoagulation with no episodes of intracranial haemorrhage. Ninety vascular events (mainly strokes) were prevented for every 1000 patient years of anticoagulation. Aspirin in a dose of $300 \mathrm{mg}$ daily had a much smaller beneficial effect, producing a $14 \%$ reduction in stroke rate.

\section{Vascular dementia}

Multi-infarct dementia and small vessel disease are increasingly being recognised as common causes of dementia and are important as they are potentially preventable. Dementia is a serious and often overlooked cause of morbidity from stroke disease. All the measures discussed above would also be expected to decrease the rates of vascular dementia, but there is little trial evidence to support this at present as none of the stroke prevention trials have used dementia as an end point. However, the importance of risk factors such as smoking ${ }^{30}$ and atrial fibrillation $^{31}$ are well recognised and at least one small trial of aspirin in patients with multi-infarct dementia has shown some benefits. ${ }^{32}$

\section{Conclusion}

Reducing the effects of vascular disease remains one of the greatest challenges facing medicine in the West. Resulting stroke and dementia are common and are serious causes of morbidity and mortality. Risk factors are now well defined making effective prevention strategies a real possibility, and many of these strategies have now been shown to be effective in large trials. It now remains to be shown that these methods are generally applicable in practice and that already stretched services can cope with the increased workload and expense. An all encompassing stroke prevention programme would now involve health education for the whole population, government measures especially aimed at reducing smoking, and changes in medical treatment, both at an individual level and in reorganising services. Such a complex package requires central organisation, and the role of government is pivotal. The undertaking by the government in the UK to reduce stroke as set out in The Health of the Nation is therefore to be welcomed. ${ }^{33}$

1 Dahlof B, Lindholm LH, Hansson L, Schersten B, Ekbom T, Wester P-O. Morbidity and mortality in the Swedish trial in old patients with hypertension (STOP-Hypertension). Lance 1991; 338: $1281-5$.

2 MRC Working Party. Medical Research Council trial of treatment of hypertension in older adults: principal results. BMJ 1992; 304: adults:

3 SHEP C.

SHEP Cooperative Research Group. Prevention of stroke by antihypertensive drug treatment in older persons with isolated systolic hypertension. $\mathcal{F} A M A$ 1991; 265: 3255-64

4 MRC Working Party. MRC trial of treatment of mild hypertension: principal results. $B M \mathcal{F} 1985$; 291: 97-104.

5 MRC Working Party. Stroke and coronary heart disease in mild hypertension: risk factors and the value of treatment. BMF 1988; 296: 1565-70.

6 Alderman MH. Blood pressure management Individualized treatment based on absolute risk and the potential for benefit. Ann Intern Med 1993; 119: 329-35.

7 Wolf PA, Dawber TR, Thomas HE, Kannel WB. Epidemiological assessment of chronic atrial fibrillation and risk of stroke: the Framingham study. Neurology 1978; 28: 973-7.

8 Wom study. Neurology 1978; 28: 973-7. Wolf PA, Abbott RD, Kannel WB. Atrial fibril-
lation as an independent risk factor for stroke: lation as an independent risk factor for stroke:

9 Wolf PA, Kannel WB, McGee DI, Meeks SL, Bharucha NE, McNamara PM. Duration of atrial fibrillation and imminence of stroke: the Framingham study. Stroke 1983; 14: 664-7.

10 Brand FN, Abbott RD, Kannel WB, Wolf PA Characteristics and prognosis of lone atria fibrillation. $\mathcal{F} A M A$ 1985; 254: 3449-53.

11 Kopecky SL, Gersh BJ, McGoon MD, et al. The natural history of lone atrial fibrillation. $N$

12 Petersen P, Godtfredsen J. Embolic complications in paroxysmal atrial fibrillation. Stroke 1986; 17: 622-6.

13 Petersen P, Boysen G, Godtfredsen J, Andersen ED, Andersen B. Placebo-controlled, randomised trial of warfarin and aspirin for prevention of thromboembolic complications in chronic atrial fibrillation: the Copenhag
study. Lancet 1989; i: 175-9.
14 The Boston area anticoagulation trial for atrial fibrillation investigators. The effect of low-dose warfarin on the risk of stroke in patients with nonrheumatic atrial fibrillatin. $N$ Engl $\mathcal{F} \mathrm{Med}$ 1990; 323: 1505-11.

15 Stroke Prevention in Atrial Fibrillation Investigators. Stroke prevention in atrial fibrillation gators. Stroke prevention in atrial
study. Circulation 1991; 84: 527-39.

16 Connolly SJ, Laupacis A, Gent M, Roberts RS, Cairns JA, Joyner C. The Canadian atrial fibrillation anticoagulation study. $\mathcal{f} \mathrm{Am}$ Coll Cardiol 1991; 18: 349-55.

17 Ezekowitz MD, Bridgers SL, James KE, et al. Warfarin in the prevention of stroke associated with nonrheumatic atrial fibrillation. $N$ Engl f Med 1992; 327: 1406-12.

18 European atrial fibrillation trial study group. Secondary prevention in non-rheumatic atrial fibrillation after transient ischaemic attack or minor stroke. Lancet 1993; 342: 1255-62.

19 Stroke Prevention in Atrial Fibrillation Investigators. Warfarin versus aspirin for prevention of thromboembolism in atrial fibrillation: Stroke Prevention in Atrial Fibrillation II Study. Prevention in Atrial Fib
Lancet 1994; 343: 687-91.

20 Landefeld CS, Goldman L. Major bleeding in outpatients treated with warfarin: incidence and outpatients treated with warfarin: incidence and
prediction by factors known at the start of prediction by factors known at the start of
outpatient therapy. Am $\mathcal{F}$ Med $1989 ; 87: 144-52$.

21 Forfar JC. A 7 year analysis of haemorrhage in patients on long-term anticoagulant treatment. Br Heart $\mathcal{f} 1979$; 42: 128-32.

22 Gurwitz JH, Goldberg RJ, Holden A, Knapic $\mathrm{N}$, Ansell J. Age-related risks of long-term oral anticoagulant therapy. Arch Intern Med 1988; 148: $1733-6$.

23 Wickramasinghe LS, Basu SK, Bansal SK. Long-term oral anticoagulant therapy in elderly patients. Age Ageing 1988; 17: 388-96.

24 Dennis M, Bamford J, Sandercock P, Warlow C. Prognosis of transient ischaemic attacks in the
Oxfordshire community stroke project. Stroke 1990; 21: 848-53.

25 Antiplatelet Trialists' Collaboration. Secondary prevention of vascular disease by prolonged antiplatelet treatment. $B M \mathscr{\jmath}$ 1988; 296: 320-31. 
26 European Carotid Surgery Trialists' Collaborative Group. MRC European Carotid Surgery Trial: interim results for symptomatic patients with severe $(70-99 \%)$ or with mild $(0-29 \%)$
$1235-43$.

27 North American Symptomatic Carotid Endarterectomy Trial Collaborators. Beneficial effect of carotid endarterectomy in symptomatic patients with high-grade carotid stenosis. $N$ Engl F Med 1991; 325: 445-53.

28 The Asymptomatic Carotid Atherosclerosis Study Group. Study design for randomized prospective trial of carotid endarterectomy for asymptomatic atherosclerosis. Stroke 1989; 20: 844-9.
29 Veterans Administration Cooperative Study. Role of carotid endarterectomy in asymptomatic carotid stenosis. Stroke 1986; 17: 534-9.

30 Homer AC, Honavar M, Lantos PL, Hastie IR, Kellett JM, Millard PH. Diagnosing dementia: Kellett JM, Millard PH. Diagnosing demen
do we get it right? $B M \mathcal{F}$ 1988; 297: 894-6.

31 Ratcliffe PJ, Wilcock GK. Cerebrovascular disease in dementia: the importance of atrial disease in dementia: the importance of atrial

32 Meyer JS, Rogers RL, McClintic K, Mortel $\mathrm{KF}$, Lofti J. Randomised clinical trial of daily aspirin treatment in multi-infarct dementia: pilot study. $\mathcal{A}$ Am Geriatr Soc 1989; 37: 549-55.

33 The Health of the Nation, a consultative documen for health in England. (CM 1523) HMSO, 1991.

\title{
Parkinson's disease
}

\author{
D Mukherjee, IR Hastie
}

Fifteen years ago the pathology of Parkinson's disease was thought to be neatly parcelled in the substantia nigra with deficiencies of the dopaminergic system in the nitro-striatal pathways. The elegant pathological simplicity previously envisioned is now known to be much more complex.

\section{Pathology}

The hallmark of Parkinson's disease was said to be the total or subtotal loss of nigral cells in the substantia nigra but this is also seen in normal ageing and is most marked in the dorsal part of the pars compacta while in idiopathic Parkinson's disease it is seen in the ventral part. Lewy bodies are seen in the brains of patients with idiopathic Parkinson's disease but their precise role is not clear. ${ }^{1}$ They are also seen in the brains of $10 \%$ of elderly controls without Parkinson's disease but are more frequent in those with the disease. ${ }^{1,2}$ Whether they are a cause or a marker of disease is not yet clear.

The previous supposition that only dopaminergic pathways are involved is known to be wrong. Abnormalities in adrenergic, cholinergic, and serotoninergic pathways have all been noted. ${ }^{3}$ The dopamine receptor has been analysed and subdivided and there are currently at least five dopamine receptor subtypes $\left(D_{1}\right.$ to $\mathrm{D}_{5}$ ) classified. Pharmacological agents with differing affinities for these receptors have been and are being developed.

1-Methyl-4-phenyl-1,2,3,6-tetrahydropyridine (MPTP) is known to produce a clinical syndrome very similar to Parkinson's disease. ${ }^{4-6}$ MPTP is converted by monoamine oxidase-B to the toxic metabolite MPP + which inactivates mitochondrial complex I. Mitochondrial complex I is one of four complexes that convert pyruvate to adenosine triphosphate in the mitochondria. MPTP accumulates in the mitochondria of dopaminergic neurons and eventually kills them. In idiopathic Parkinson's disease levels of complex I are known to be depressed and mitochrondrial defects have been reported in the brains, ${ }^{7,8}$ platelets $^{9}$ and muscles ${ }^{10}$ of patients with Parkinson's disease. No MPTP-like substance has yet been identified in the environment but this pathological model involving an environmental cause as a potent factor is a tempting one.

\section{Clinical features}

Other features known to be of significance in addition to the classic triad of tremor, bradykinesia, and ridigity are impaired postural reflexes, shuffling gait,

Division of Geriatric Medicine, St George's Hospital Medical School, London SW17 ORE, UK

D Mukherjee

IR Hastie

\section{Correspondence to IR Hastie}

Accepted 8 June 1994 progression of physical signs, asymmetry at onset and response to dopaminergic agents. Dysarthria is common and dysphagia is increasingly recognised as a problem. Many methods are currently used to evaluate the degree of disability and severity of Parkinson's disease. ${ }^{11-14}$

Other conditions may share features with idiopathic Parkinson's disease and need to be excluded. These include progressive supranuclear palsies, multisystem atrophy, exposure to toxins, Wilson's disease and Alzheimer's disease with extrapyramidal symptoms. 